\title{
Sensibilidad estacional en población de altas latitudes y su relación con variables de adaptación y estilo de organización temporal del trabajo
}

\section{Seasonal sensitivity in a high-latitude population and its relationship to adaptation variables and style of seasonal work organization}

\section{Sensibilidade estacional em população de altas latitudes e sua relação com variáveis} de adaptação e estilo de organização temporal de trabalho

\author{
Iben Gueichatureo Asencio ${ }^{1}$, ORCID 0000-0002-6871-0813 \\ Felipe Loezar Tillería ${ }^{2}$, ORCID 0000-0002-5311-2589 \\ Viviana Mellado Quiroz ${ }^{3}$, ORCID 0000-0003-0588-5410 \\ Carlos Vera Vega ${ }^{4}$, ORCID 0000-0001-8041-2838 \\ Camila Jelincic Vasquez, ${ }^{5}$, ORCID 0000-0002-5417-3041 \\ Cristian Nuñez Espinoza ${ }^{6}$, ORCID 0000-0002-9896-7062 \\ Claudia Estrada-Goic ${ }^{7}$, ORCID 0000-0002-9982-2025 \\ 1234567 Universidad de Magallanes, Chile
}

\begin{abstract}
Resumen: El trabajo estudió la frecuencia de sensibilidad estacional en una muestra de población austral chilena, alta latitud sur y su relación con actividades laborales desarrolladas en diferentes turnos de trabajo y con variables de adaptación general. Un total de 465 personas participaron respondiendo instrumentos midiendo estacionalidad (SPAQ), felicidad subjetiva (Escala de Oxford), adaptación social (Bell) y ansiedad y depresión (MASQE30). Los resultados principales indican que la frecuencia de sensibilidad estacional es alta, su nivel declarado en promedio es moderado, en su límite superior, y es evaluada como de impacto negativo significativo en la vida diaria. Respecto de los patrones estacionales, el más numeroso fue el de verano, que indica que las personas perciben beneficios con el aumento de luz, lo que correlaciona negativamente con la felicidad. Los análisis según turno de trabajo mostraron perfiles diferenciales para cada uno. Se discuten estos resultados en torno a su importancia para el desarrollo de políticas públicas asociadas a la salud física y mental de poblaciones australes.
\end{abstract}

Palabras clave: sensibilidad estacional; patrones; adaptación social; turno de trabajo; felicidad.

\section{(c) $)$ EY}

Esta obra está bajo una licencia de Creative Commons Reconocimiento 4.0 Internacional 
Abstract: This study is intended to determine the frequency of seasonal sensitivity in a sample of a Chilean high latitude (extreme southerly) population, and its correlation with labor activities involved in several types of shift work, with variables of general adaptation. A total of 465 participants took part in answering questionnaires to measure seasonality impacts through SPAQ (Seasonal Pattern Assessment Questionnaire), subjective happiness (Oxford scale), social adaptation (Bell), and anxiety and depression (MASQE30). The principal results indicate that the frequency of seasonal sensitivity in this sample was high, in a moderate level near the upper limit, and this is considered to have a significantly negative impact on daily life. Regarding seasonal patterns, the most common effect was in the summer profile, suggesting that people perceive benefits with the increase in sunlight, which correlates negatively with happiness. The analysis according to work-shift shows differential profiles for each one. These results are discussed relative to the importance for development of public policies to further the physical and mental health of extreme-southern populations.

Keywords: seasonal sensitivity; pattern; social adaptation; work shift; happiness.

Resumo: Este trabalho estudou a frequência de sensibilidade sazonal em uma amostra de população austral chilena, nas altas latitudes do sul, e sua relação com atividades laborais desenvolvidas em diferentes turnos de trabalho e com variáveis de adaptação geral. Um total de 465 pessoas participaram respondendo instrumentos que mediam a sazonalidade (SPAQ), a felicidade subjetiva (Escala de Oxford), a adaptação social (Bell) e a ansiedade e a depressão (MASQE30). Os principais resultados indicam que a frequência de sensibilidade estacional é alta, seu nível declarado em média é moderado em seu limite superior e é avaliado como de impacto negativo significativo na vida diária. Com respeito aos padrões estacionais, o mais numeroso foi o do verão, que indica que as pessoas percebem benefícios com o aumento da luz, o que se relaciona negativamente com a felicidade. As análises de acordo com o turno de trabalho mostraram perfis diferenciais para cada um deles. Discutemse esses resultados em torno a sua importância para o desenvolvimento de políticas públicas associadas a saúde física e mental das populações austrais.

Palavras-chave: sensibilidade estacional; padrões; adaptação social; turno de trabalho; felicidade.

Recibido: 22/11/2020

Aceptado: 04/10/2021

Cómo citar:

Gueichatureo, I., Loezar, F., Mellado, V., Vera, C., Jelincic, C., Nuñez, C., Estrada-Goic, C. (2021). Sensibilidad estacional en población de altas latitudes y su relación con variables de adaptación y estilo de organización temporal del trabajo. Ciencias Psicológicas, 15(2), e2344. doi: https://doi.org/10.22235/cp.v15i2.2344

Correspondencia: Claudia Estrada-Goic, Universidad de Magallanes, Chile. E-mail: claudia.estrada@umag.cl 
El ser humano ha mostrado una gran capacidad de adaptación a diversos ambientes naturales no sin que este proceso tenga impacto en el bienestar y salud de las personas. Existen numerosas variables ambientales que condicionan el funcionamiento psicológico de las personas y su adaptación social (Argyle, 1992; Barrientos, 2005; Diener, 1994; Diener, Suh, Lucas \& Smith, 1999). Dentro de los condicionantes físicos la exposición a los cambios en la insolación debidos a las estaciones del año ocupa un lugar relevante (Wehr et al., 2001), en particular en zonas en las que los cambios de luminosidad son más extremos (altas latitudes). Los cambios en las horas de luz han mostrado tener impacto en aspectos centrales de la adaptación social de los seres humanos, afectando sus hábitos, estados de ánimo y percepción de bienestar (Malbos, 2020; Rohan, Sigmon \& Dorhofer, 2003). Los cambios en la insolación impactan a nivel biológico en los niveles de serotonina y cortisol, con los consecuentes cambios comportamentales que los acompañan (Gatón, González \& Gaviria, 2015; Labbé, Veliz, Saavedra, Arab \& Martorell, 2011; Lambert, Reid, Kaye, Jennings \& Esler, 2002; Lansdowne \& Provost, 1998). Los principales cambios que se observan debido a estos cambios han sido consolidados en los conceptos de "estacionalidad" o de "sensibilidad estacional". Ambos aluden a las variaciones estacionales en el humor y el comportamiento, así como también en otros aspectos de la vida como la actividad social, el aumento de peso, el deseo sexual y la energía vital (Rohan et al., 2003; Rosen et al. 1990; Rosenthal, 1993; Rumble et al. 2018; Stumpf \& Privette, 1989; Wirz-Justice, Graw, Kraüchi \& Wacker, 2003). En el invierno se generan condiciones para que la disminución de la luz solar se manifieste en sintomatología depresiva y ansiosa, en lo que popularmente ha sido denominado inviernos azules (winter blues), que puede ser definido como el sentimiento de sentirse triste o infeliz asociado con la experiencia del frío y oscuridad del invierno (Gatón et al., 2015). Un derivado psicopatológico de este fenómeno es el Trastorno Afectivo Estacional (TAE, SAD: Seasonal Affective Disorder) descrito por primera vez por Rosenthal et al. (1984, 1986), quienes intentaron comprender el fenómeno de la experiencia depresiva de los inviernos y su remisión espontanea en primavera-verano y postularon que la disminución en la insolación era la causa de ese malestar. Sus síntomas principales son la tristeza, ansiedad, irritabilidad, anhedonia, astenia y dificultad para concentrarse (WirzJustice et al., 2003). Estudios realizados en numerosos países indican que la prevalencia del malestar asociado a la estacionalidad es alta, bordeando el $85 \%$ (Dam, Jakobsen \& Mellerup, 1998; Kasper, Wehr, Bartko, Gaist \& Rosenthal, 1989). La sensibilidad estacional ha mostrado conexiones con numerosas enfermedades biológicas, tales como la angina, diabetes, hipertensión, colesterol alto, entre otras; afectando el desarrollo satisfactorio de la vida adulta de quienes la padecen, así como también en el plano interpersonal, familiar y laboral (Basnet, Rokaya, Bhattarai \& Münzbergová, 2016; Garbazza \& Benedetti, 2018; Gatón et al., 2015; Joiner, Catanzaro, Laurent, Sandin \& Blalock, 1996; Kasper et al., 1989; Kawasaki et al., 2018; Oyane, Reidun, Pallesen, Holsten \& Bjorvatn., 2010; Saarijärvi, Lauerma, Helenius \& Anglé, 1999). Aunque existen descripciones del fenómeno en manuales nosológicos, tales como el DSM-5 (American Psychiatric Association, 2013), el instrumento más utilizado en la investigación del fenómeno es el cuestionario de medición de patrones estacionales (SPAQ; Rosenthal, Bradt \& Wehr, 1984). Este instrumento permite calcular un índice de estacionalidad general (GGS) y determinar las estaciones del año en la que las personas indican mayores cambios en sus hábitos generales, pudiendo establecerse la presencia de un patrón de invierno y/o de verano. El patrón estacional general se define por 
la presencia de afectación por los cambios de luz, tanto en verano como en invierno, al menos en un mes; el patrón estacional de verano implica la percepción de que los cambios de luz en el verano afectan positivamente (sintiéndose mejor cuando hay más luz); por último, el patrón estacional de invierno tiene relación con verse afectado por los cambios de luz en el invierno (sintiéndose peor cuando hay menos luz) (Magnusson, 1996).

La relación entre la sensibilidad estacional y variables sociodemográficas, tales como la edad, nivel educacional, sexo, etc., no ha mostrado diferencias concluyentes (Bjorvatn, Saxvig, Waage \& Pallesen, 2020; Gatón et al., 2015; Magnusson, 2000; Rosen et al., 1990). Su vínculo con las latitudes extremas y el cronotipo individual también han sido objeto de controversia, aunque existen evidencias de la importancia de estas dos variables. Las poblaciones de latitudes altas, particularmente norte, han mostrado alta prevalencia de sensibilidad estacional sobre todo en su patrón invernal (Kegel, Dam, Ali \& Bjerregaard, 2009). Sin embargo, existen diferencias individuales en la forma en la que las personas ajustan sus relojes biológicos a los cambios de oscuridad y luz que se producen en un día, lo que ha sido llamado cronotipo (Roenneberg, Pilz, Zerbino \& Winnebeck, 2019). La evaluación del cronotipo es la variación circadiana individual que separa a las personas en tres tipos diferentes: matutino (madrugador), intermedio y vespertino (trasnochador), según el momento del día en el que una persona se siente más alerta (Adan et al., 2012; Kivelä, Papadopoulos \& Antypa, 2018). Estudios recientes indican que existe una relación positiva entre el cronotipo vespertino y la presencia de sensibilidad estacional (Bjorvatn et al., 2020).

En cuanto a las variables psicológicas, existen estudios en población adulta de la relación entre la sensibilidad estacional y el bienestar general (ansiedad, sentimientos negativos, percepción de felicidad, etc.) (Kasper et al., 1989). Sin embargo, el efecto de la privación de luz natural por razones laborales y la sensibilidad estacional no ha sido abordada. Los trabajadores que desarrollan actividades ya sea únicamente de día (diurno), de noche (nocturno) o alternados (a veces de día y a veces de noche) podrían presentar perfiles diferenciales de sensibilidad estacional. Los trabajadores no diurnos sufren la desregulación de la vida normativa, desde un punto de vista social, afectando su vida sociofamiliar y psicológica, presentándose en parte de ellos sintomatología depresiva sin contar con el factor estacional (Goodrich \& Weaver, 1998). El trabajo nocturno se vincula con la disminución de la productividad y el aumento del riesgo de accidentes graves cuando este se ha extendido por periodos largos (Nielsen et al., 2018). Trabajadores con turnos alternados han mostrado aumento del estrés y mayores dificultades para adaptarse adecuadamente al trabajo, en particular cuando se trata de actividades que implican el cuidado de otras personas (NabeNielsen, Tuchsen, Christensen, Garde \& Diderichsen, 2009). En términos amplios, las investigaciones desarrolladas desde hace más de 60 años en personas que trabajan en turnos no diurnos (nocturnos o alternados) muestran consistentemente indicadores de menoscabo en su salud física y psicológica, mostrando una mayor prevalencia de cuadros ansiosos y distímicos, emociones negativas, y bajo bienestar y felicidad percibida (Bohle, Willaby, Quinlan \& McNamara, 2011; Fischer et al., 2019). Basado en la convergencia de los efectos de la sensibilidad estacional y el trabajo por turnos, es posible hipotetizar que ambas interactúan intensificando los efectos sobre el afecto positivo (actividad y entusiasmo) y el afecto negativo (como el nerviosismo, el pesimismo y la baja autoestima), la percepción de felicidad subjetiva y, en términos globales, la adaptación social (Bharvad, 2015; Buckby, Cotton, Cosgrave, Killackey \& Yung, 2008; Jiménez \& López-Zafra, 2011; Rosenthal, 1993; 
Watson, Clark \& Carey, 1988). Finalmente, la adaptación social puede ser comprendida como un proceso que busca mantener o alcanzar el equilibrio entre las necesidades del yo como organismo y su encuentro con el entorno social o físico (Bell, 1934; Birchwood, Smith, Cochrane, Wetton \& Copestake, 1990; Lyubomirsky, 2008; Vendrik, 2013). En el entorno social se puede encontrar la demanda laboral del trabajo en diferentes horarios (turnos) y en el físico las condiciones de insolación del lugar en el que se vive. La adaptación social incluye un elemento subjetivo que se manifiesta en la percepción del logro o no de un estado de felicidad (Lyubomirsky \& Lepper, 1999; Lyubomirsky, Sheldon \& Schkade, 2005).

En resumen, la estacionalidad puede ser considerada una variable ambiental (atmosférica), con influencia en el equilibrio psicológico (Goikolea, Miralles, Cabré, Vieta \& Bulbena, 2003). Sus efectos son amplios e incluyen consecuencias psicológicas y psicopatológicas que afectan el bienestar percibido. Los efectos de la estacionalidad pueden ser paradojales, pero afectan la adaptación general de las personas. Aunque se conocen algunos de los factores moderadores de sus efectos, no existen estudios que se ocupen de su relación con la exposición a la luz delimitada por las actividades laborales. El presente estudio, por consecuencia, tiene como objetivo explorar la frecuencia en que se presenta la sensibilidad estacional, su severidad, patrones preferenciales y su relación con emociones positivas y negativas, felicidad percibida y adaptación social en una muestra de población de alta latitud sur $\left(53^{\circ} 9^{\prime} 45.72^{\prime \prime} \mathrm{S}, 70^{\circ} 54^{\prime} 29.16^{\prime \prime} \mathrm{W}\right)$, que realizan trabajos diurnos, nocturnos y alternados.

\section{Método}

\section{Participantes}

Participaron un total de 465 personas voluntarias, seleccionadas mediante muestreo no aleatorio por conveniencia. El criterio de inclusión fue ser mayor de edad, ser chileno y encontrarse activo laboralmente. Un $54.2 \%$ de la muestra indicó ser trabajador de turno diurno (ejerce sus actividades en un horario entre las 08:00 y las 19:30), 31.3 \% declaró trabajo de turnos alternados (algunos días de la semana durante jornada diurna y otros días jornada de noche) y el $4.53 \%$ restante trabaja en turno nocturno (en horario después de las 19:30 y antes de las 08:00). Respecto al estado civil, la distribución en los tres tipos de turnos de trabajo es similar: cerca de un $50 \%$ de cada submuestra se declara soltero o sin una relación estable, un $43 \%$ casado o con un vínculo estable, mientras que el $7 \%$ restante es divorciado, separado o viudo. La moda de nivel educacional fue estudios superiores completos $(67 \%)$, seguido de estudios superiores incompletos $(22 \%)$ la que resultó similar para cada turno de trabajo. Lo mismo ocurre con el nivel de ingreso, donde el $92 \%$ indicó ingresos igual o inferior a 700 USD mensuales. En cuanto a la distribución según género, un $32 \%$ masculino y un $68 \%$ de género femenino. La distribución según turno fue diurno (78 \% femenino), alternado (62\% femenino) y nocturno (56\% femenino). Se realizó una diferenciación entre quienes se consideran magallánicos con un $76 \%$ en comparación a un $23.3 \%$ quienes se sienten pertenecientes a otro grupo. El rango de edad de los participantes fue de 17 a 88 años $(M=34.3 ; D E=12.9)$. 


\section{Instrumentos}

La totalidad de los instrumentos se utilizó en versiones de español chileno que han seguido procesos de validación de contenido y constructo, y cuentan con normas regionales.

Medidas sociodemográficas. Se midieron las variables: género, edad, ciudad de origen, pertenencia a un grupo, tiempo de residencia en la región, estado civil, estudios formales, actividad, ingresos y sistemas de trabajo (diurno, alternado o nocturno). Este instrumento ha sido testeado previamente para establecer su confiabilidad con el método testretest.

Cuestionario de estacionalidad (SPAQ; Goikolea et al., 2003). Tiene como objetivo medir la sensibilidad de las personas frente a los cambios de luminosidad, evaluando la percepción de cambio en el estado de ánimo frente a las estaciones y sintomatología depresiva, tal como cambios en la alimentación, estado anímico, cambios en la sociabilidad y horas de sueño. Se usa preferentemente como instrumento de cribaje con buenos indicadores de calidad psicométrica (Adan, Natali \& Fabris, 2006). El instrumento consta de secciones. La primera compuesta por 6 ítems explora la sensibilidad del individuo a las variaciones estacionales en relación al sueño, actividad social, bienestar general, peso, apetito y energía. Las opciones de respuesta varían desde 0 (sin cambios) hasta 4 (cambio extremo). La suma construye la puntuación global de estacionalidad (GGS) que tiene un máximo de 24 puntos, siendo los puntajes mayores los que indican mayor sensibilidad estacional. Los puntajes menores a 10 se categorizan como "sin sensibilidad estacional", los que se encuentran entre 9 y 12-14 como "sensibilidad estacional" (subsíndrome de TAE) y mayores a 15 puntos son interpretados como posible presencia de TAE. La segunda sección tiene 10 ítems, que se evalúan para cada mes del año (ej: "tengo más actividad social”), incluyendo la opción "en ninguno". Para determinar el patrón de verano se evalúa la declaración de percepción de sensibilidad a los cambios de luz en los meses de verano, el patrón de invierno cuando la sensibilidad a los cambios es sindicada como de ocurrencia en los meses de dicha estación; y el patrón mixto, cuando el participante indica simultáneamente sensibilidad en verano y en invierno.

Escala de Felicidad Subjetiva Oxford (Lyubomirsky \& Lepper, 1999). Esta escala tiene como objetivo obtener una medida global de felicidad subjetiva mediante enunciados en los que la persona se evalúa a si mismo o bien se compara con quienes le rodean. Se compone de cuatro ítems ("En general me considero...", "En comparación con la mayoría de mis iguales, me considero", "Algunas personas son muy felices en general. Disfrutan de la vida independientemente de lo que suceda, disfrutan al máximo todo ¿Hasta qué punto le describe esta caracterización?", "Por término general, algunas personas no son muy felices. Aunque no se encuentran deprimidas, nunca parecen estar felices como podrían. ¿Hasta qué punto le describe esta caracterización?"), los cuales se responden mediante una escala tipo Likert de 7 puntos. Puntajes altos (luego de invertir el ítem 4 que es indirecto) señalan alta felicidad percibida. El alfa de Cronbach para esta muestra es $=.77$.

Cuestionario de adaptación social para adultos (Bell, 1934). Tiene como objetivo medir el estado general de adaptación adulta utilizando cinco dimensiones representativas de la adaptación adulta: familiar, social, laboral, de la salud y emocional. Para el presente estudio se utilizó la subescala de adaptación social, de 33 ítems (con una escala de respuesta: si, no, no sé), que hace referencia al establecimiento y mantención de relaciones interpersonales adecuadas, tanto en el plano íntimo como en el ambiente social inmediato en 
el que un individuo se desarrolla (familia, trabajo, comunidad). Puntajes altos en esta escala indican dificultades para una adaptación social satisfactoria mostrando dificultades para desarrollar un estado de pertenencia satisfactorio con el entorno social percibiendo insatisfacción general respecto a las esferas sociales en las que se desempeña (Bell, 1934; Jiménez \& López-Zafra, 2011). Utilizando la fórmula de Spearman-Brown, el autor original reportó $\rho=.80$ para adaptación social. El alfa de Cronbach para esta muestra es $=.68$. Algunos ejemplos de ítems son: “¿Se sentiría usted muy intimidado si fuera el encargado de lanzar una idea para organizar una discusión en un grupo?”; “Se encuentra intimidado en presencia de personas que admira mucho, pero a las que no conoce demasiado?”; “Se siente intimidado o cohibido al solicitar un trabajo?".

Cuestionario de Ansiedad y Humor (MASQE30; versión española de González \& Ibáñez, 2018). Diseñado para medir tres dimensiones del modelo tripartito de la ansiedad y la depresión, estas son: el Afecto Negativo (AN), el Afecto Positivo (AP) y la Ansiedad Somática (AS). Esta versión es en español de Canarias, que es altamente afín al español latinoamericano, reporta adecuados indicadores de validez de constructo, mediante análisis factoriales exploratorio y confirmatorio, así como validez convergente, discriminante y predictiva con ansiedad y depresión evaluadas con cuestionarios. Sus coeficientes de consistencia interna que oscilan entre .88 y .92 y una estabilidad temporal que va desde .47 a .68. Se utilizaron las dos primeras dimensiones que alcanzaron alfas de Cronbach para esta muestra $=.85$ y .79 respectivamente (cada una compuesta de 10 ítems). Ejemplos de ítems de AN: "Regularmente me siento insatisfecho con todo", "Usualmente me siento irritable"; y de AP: "Por lo general siempre me divierto mucho", "A menudo me siento bien conmigo mismo/a".

\section{Procedimiento}

La participación fue voluntaria. Las personas fueron contactadas en sus lugares de estudio y trabajo durante un período aproximado de cuatro meses (período prepandemia). La dificultad de acceso a trabajadores de turnos diferente a los diurnos exigió que dichas personas fueran invitadas a participar (invitación pública y vía redes sociales) y luego se les ofreciera contestar a los instrumentos en un lugar de su conveniencia. Aproximadamente un $50 \%$ de los participantes recibieron dichos cuestionarios en su lugar de trabajo por su solicitud expresa. La aplicación en terreno fue realizada por un equipo de dos asistentes de investigación entrenados en esta tarea. Previa participación, se les solicitó la lectura y firma de un consentimiento informado. Dicho consentimiento, aprobado por el Comité de Ética institucional, informaba respecto a los derechos del participante en el estudio incluyendo información sobre el tratamiento de sus datos personales. Los instrumentos fueron autoaplicados y se contestaron sus dudas durante el proceso. Al finalizar el estudio los participantes recibieron un informe personal de sus resultados, como forma de retribuir su participación, en el que se incluían sus resultados en las variables centrales del estudio. La elaboración y entrega del estudio se realizó mediante una modalidad de doble ciego para que sus datos personales no pudiesen ser vinculados a sus resultados más allá del folio común.

\section{Diseño}

No experimental, transversal, correlacional. 


\section{Plan de análisis}

Para cumplir el objetivo de explorar la frecuencia en que se presenta la sensibilidad estacional, su severidad y patrones estacionales se realizaron análisis descriptivos de frecuencia. Siguiendo las instrucciones del instrumento se calculó la puntuación global de estacionalidad (GGS), así como también la presencia de patrones de sensibilidad estacional verano, invierno o mixto. La severidad también requirió de análisis descriptivos realizados sobre el ítem que evalúa este aspecto del fenómeno, así como también en aquellos que refieren a sus efectos seis diferentes aspectos, tales como el sueño y el bienestar.

La relación de los índices de sensibilidad estacional con las emociones positivas y negativas, felicidad percibida y adaptación social requirió de análisis correlacionales de Spearman, ya que el supuesto de normalidad no se cumplió para la variable felicidad. Para el objetivo de comparar los tres tipos de turnos de trabajo (diurno, alternado y nocturno) se utilizaron ANOVAs univariadas.

\section{Resultados}

Del total de participantes, un $37.5 \%$ señala presentar una sensibilidad estacional marcada, mientras que un $36 \%$ de ellos indica que este fenómeno es un problema para ellos. De entre estos últimos, un $26.7 \%$ indica que su efecto es leve, un $39 \%$ lo declara como moderado, mientras que el $34.3 \%$ restante indica que es importante o severo. No se observó una distribución diferente según turno de trabajo $(\chi(2, \mathrm{n}=339)=0.73$, n.s. $)$. El promedio global de estacionalidad (GGS) se sitúa en el rango de subsíndrome de TAE (tabla 1). Al comparar el puntaje de GGS según turno de trabajo se aprecia que los participantes que trabajan en horario diurnos reportan mayor presencia de estacionalidad que aquellos de turnos mixtos o nocturnos (tabla 2).

Tabla 1.

Medias, desviaciones estándar y correlaciones de las variables del estudio

\begin{tabular}{|c|c|c|c|c|c|c|c|c|}
\hline \multirow{2}{*}{ Variables } & \multirow[b]{2}{*}{$M$} & \multirow[b]{2}{*}{$D E$} & \multicolumn{6}{|c|}{ Correlaciones parciales } \\
\hline & & & 2 & 3 & 4 & 5 & 6 & 7 \\
\hline $\begin{array}{l}\text { 1. Estacionalidad } \\
\text { general }\end{array}$ & 9.75 & 5.39 & $.43 * *$ & $22 * *$ & $\begin{array}{c}- \\
.18^{* * *}\end{array}$ & $.14 * *$ & $.24 * *$ & .23 \\
\hline 2. Patrón de invierno & - & - & - & $.21 * *$ & $\begin{array}{c}- \\
.21^{*} *\end{array}$ & .07 & $.11^{*}$ & $.22 * *$ \\
\hline 3. Patrón de verano & - & - & & - & .07 & $.16^{* *}$ & .04 & .05 \\
\hline 4. Felicidad & 5.11 & 1.50 & & & - & .02 & $\begin{array}{c}- \\
.17^{* *}\end{array}$ & $.49 * *$ \\
\hline 5. Adaptación general & 20.61 & 6.40 & & & & - & .01 & .01 \\
\hline 6. Afecto negativo & 2.84 & 1.86 & & & & & - & ${ }^{-} \cdot 16^{* *}$ \\
\hline 7. Afecto positivo & 4.79 & 1.10 & & & & & & - \\
\hline
\end{tabular}

Notas: * Correlaciones significativas al $p<.05 * *$ Correlaciones significativas al $p<.01 * * *$ 
Tabla 2.

Medias según tipo de turno

\begin{tabular}{lcccccccc}
\hline \multirow{2}{*}{ Variables } & \multicolumn{2}{c}{ Diurno } & \multicolumn{2}{c}{ Alternado } & \multicolumn{2}{c}{ Nocturno } & & \\
\cline { 2 - 7 } & $M$ & $D E$ & $M$ & $D E$ & $M$ & $D E$ & $F$ & $p$ \\
\hline Estacionalidad general & $10.50_{\mathrm{a}}$ & 5.04 & $9.15_{\mathrm{b}}$ & 5.66 & $8.11_{\mathrm{b}}$ & 5.63 & 6.43 & .001 \\
Felicidad & $4.91_{\mathrm{a}}$ & 1.60 & $5.27_{\mathrm{b}}$ & 1.30 & $5.61_{\mathrm{b}}$ & 1.38 & 6.61 & .001 \\
Adaptación general & $22.98_{\mathrm{a}}$ & 5.02 & $17.57_{\mathrm{b}}$ & 6.51 & $18.08_{\mathrm{b}}$ & 7.34 & 44.81 & .001 \\
Afecto negativo & $2.77_{\mathrm{b}}$ & 1.12 & $2.80_{\mathrm{b}}$ & 1.07 & $3.22_{\mathrm{a}}$ & 1.15 & 4.32 & .014 \\
Afecto positivo & $4.61_{\mathrm{a}}$ & 1.03 & $5.00_{\mathrm{b}}$ & 1.13 & $5.04_{\mathrm{b}}$ & 1.14 & 7.63 & .001 \\
\hline
\end{tabular}

Con respecto a la frecuencia de la presencia de patrones estacionales, un $39.4 \%$ de los participantes califica como perfil de invierno; mientras que los que presentan perfil de verano fueron un $71 \%$. Los que comparten ambos perfiles combinados alcanzan a un $31.8 \%$. Según sus turnos de trabajo, mientras que el perfil mixto se distribuye equitativamente entre los tres tipos de turnos, los perfiles de verano e invierno muestran diferencias. La distribución de los perfiles de verano está presente en el $80 \%$ de los turnos diurnos, en un $70 \%$ de los nocturnos y en un $58 \%$ de los que trabajan en turnos alternados. En el caso de los perfiles de invierno, los participantes que trabajan en turnos diurnos $(49 \%)$ presentan un porcentaje mayor de este patrón que aquellos que realizan sus actividades en turno alternado (34\%) o nocturno (29\%). En otros términos, la proporción de perfiles de verano y de invierno es más alta en trabajadores diurnos, que son quienes regularmente se exponen a los cambios de insolación.

Las áreas que son percibidas como las más afectadas por la sensibilidad estacional se encuentran en la tabla 3. En términos generales, los participantes consideran como evidentes (marcado o muy marcado) los cambios en el nivel de energía (33\%), el apetito $(21.3 \%$ ) y la duración del sueño (20.7 \%). Según turnos, al comparar las proporciones de acuerdo, aquellos que trabajan en turnos diurnos indican que su actividad social, ánimo y nivel de energía se ven afectados por los cambios estacionales. Proporcionalmente, los trabajadores en turno nocturno indican menos afectación de su ánimo y vida social, pero más de su apetito. En el caso de aquellos que trabajan en turnos alternados, son menos los que indican que existe un efecto negativo sobre su energía.

Las correlaciones entre promedio global de estacionalidad (GGS) y las otras variables del estudio pueden ser observadas en la tabla 1. Tanto la percepción de felicidad como la adaptación social muestran relaciones significativas indicando que el aumento de la estacionalidad se vincula con un aumento de las dificultadas para lograr una adaptación social satisfactoria y una disminución de la felicidad, y viceversa. En coherencia con el resultado 
anterior, el aumento de la estacionalidad también se acompaña del aumento de afectos negativos y disminución de afectos positivos.

Las diferencias según turno de trabajo se encuentran consignadas en la tabla 3. Al comparar los tres turnos (diurno, nocturno y alternado) se evidencian diferencias en las otras cuatro variables propuestas. El promedio de felicidad percibida y de afectos positivos es menor en el turno diurno, mostrando además menor adaptación social. El turno nocturno es el que reporta mayor presencia de sentimientos negativos. El alternado no se diferencia significativamente de forma consistente de ninguno de los otros dos turnos, mostrándose similar a cada uno de ellos en diferentes variables.

Tabla 3.

Porcentajes de grado de cambio según turno de trabajo

\begin{tabular}{|c|c|c|c|c|c|c|c|c|c|c|c|c|c|c|c|}
\hline & \multicolumn{3}{|c|}{ No cambia } & \multicolumn{3}{|c|}{ Cambia poco } & \multicolumn{3}{|c|}{ Cambio moderado } & \multicolumn{3}{|c|}{ Cambio marcado } & \multicolumn{3}{|c|}{ Cambio muy marcado } \\
\hline & G1 & G2 & G3 & G1 & G2 & G3 & G1 & $\mathrm{G} 2$ & G3 & G1 & G2 & G3 & G1 & G2 & G3 \\
\hline Duración sueño & 21 & 24 & 16 & 29 & 34 & 30 & 30 & 22 & 32 & 15 & 13 & 15 & 05 & 07 & 07 \\
\hline Actividad social & 22 & 45 & 31 & 25 & 24 & 28 & 28 & 21 & 27 & 19 & 08 & 09 & 07 & 02 & 05 \\
\hline Ánimo & 15 & 29 & 24 & 34 & 29 & 33 & 29 & 28 & 23 & 16 & 08 & 11 & 07 & 05 & 09 \\
\hline Peso & 11 & 27 & 10 & 31 & 29 & 38 & 43 & 26 & 33 & 11 & 12 & 13 & 05 & 06 & 06 \\
\hline Apetito & 15 & 30 & 16 & 31 & 25 & 36 & 35 & 18 & 31 & 15 & 19 & 12 & 05 & 07 & 06 \\
\hline Nivel de energía & 09 & 24 & 18 & 20 & 24 & 23 & 33 & 18 & 32 & 24 & 23 & 14 & 14 & 11 & 13 \\
\hline
\end{tabular}

Notas: G1 = Turno Diurno; G2 = Turno nocturno; G3 = Turno alternado.

\section{Discusión y conclusión}

El presente estudio tuvo como objetivo determinar la frecuencia de la sensibilidad a los cambios estacionales de la insolación en una muestra de adultos trabajadores que habitan en alta latitud sur y que desarrollan su trabajo en turnos ya sean estos diurnos, nocturnos o alternados. El resultado principal indica que más de un tercio de los trabajadores señalan presentar sensibilidad estacional en niveles que afectan su adaptación general y una cifra similar declara que los efectos de los cambios en luminosidad son problemáticos para su vida cotidiana. Aunque el promedio general de la muestra se sitúa en el rango estacionalidad moderada en su límite superior, cerca del puntaje que se considera TAE, fueron los trabajadores en turno diurno quienes declararon mayor afectación.

Aunque el instrumento utilizado ha sido criticado por su sobrerrepresentación de este síndrome, el puntaje global indica la presencia de una sensibilidad estacional franca. Una explicación a este fenómeno está en el factor geográfico, ya que los participantes viven cerca de lo que es considerado alta latitud sur (53). Por otra parte, el resultado que indica que son los trabajadores diurnos los que indican mayor sensibilidad estacional, puede explicarse en que son los que presencian los cambios de la insolación de forma más rotunda durante su jornada activa. En algunos días la variación de pérdida o ganancia de minutos de luz en alta latitud puede llegar a ser de 5 minutos, lo que en una semana acumulada representa un 
fenómeno sensible. Los trabajadores nocturnos suelen dormir parte importante del día al igual que los que tienen turnos alternados que, además, tienen periodos de insolación más esporádicos y, por lo tanto, podría hipotetizarse que la percepción de los cambios es menos regular. Otra posible explicación podría estar en las diferencias en la personalidad. Se han descrito tipologías circadianas diferentes para los matutinos y vespertinos, los que no presentan el mismo nivel de activación (acrofase) en lo fisiológico (Matthews, 1998).

Respecto a los patrones preferenciales, el perfil de verano resultó ser más frecuente que el de invierno. Aunque los trabajadores no significan a los cambios debidos al aumento de la luz como negativos, son una manifestación evidente de sensibilidad por cuanto revelan la presencia de una vulnerabilidad psicológica. Lo anterior indica que estos trabajadores se ven fuertemente influenciados por las condiciones lumínicas del ambiente, lo que tendría como consecuencia una inestabilidad en el bienestar psíquico, siendo más susceptibles a los cambios de exposición a las horas de luz de acuerdo con la estación (Rihmer, 1980). La interpretación de este resultado se ratifica en la relación negativa observada entre felicidad, adaptación social y estacionalidad. Las personas que presentan sensibilidad estacional, al ser susceptibles a los cambios de insolación, muestran cambios fisiológicos que se manifiestas en dificultades para regular los estados de ánimo, motivación y energía (Tam et al., 1997). Como consecuencia, la mayor estacionalidad se vincula negativamente tanto con la percepción de felicidad como de adaptación social. Aunque es posible plantear que exista una relación causal entre ellas en la que la variable causa sería la sensibilidad estacional, se requieren de otros estudios que permitan afirmar la dirección de dicho efecto.

Por último, el que los trabajadores diurnos se presenten como menos felices, menos adaptados y menos positivos que los otros dos grupos de trabajadores, podría obedecer a que ellos pueden percibir las presiones de la vida cotidiana, mientras que los otros operan durante un periodo en que la comunidad, su actividad y las demandas que la acompañan se hallan mayormente suspendidas (Angosto, 1992; Fischer et al., 2019). En cuanto a los turnos alternados, esta condición, según ha sido reportado en la literatura, les permitiría una mejor adaptación familiar (Guérin \& Durrmeyer, 1973). Comprender de manera más profunda estas diferencias requiere de la inclusión de otras variables potencialmente moderadoras, tales como el tiempo de permanencia en el turno de trabajo, y también de otras variables sociodemográficas, como la densidad de la red social de apoyo.

Ya que una debilidad evidente del presente estudio es la utilización de una muestra no aleatoria, afectando la representación poblacional de la misma, futuras investigaciones debiesen orientarse a muestras aleatorias que abarquen a un mayor número de habitantes y que equiparen variables sociodemográficas potencialmente relevantes, como el género y la composición familiar. El estudio de este fenómeno aparentemente tan frecuente en la población general local debiese avanzar en la determinación de variables moderadoras, como el punto del ciclo laboral en el que se encuentra cada persona adulta y su evolución longitudinalmente.

Las implicaciones teóricas de estos hallazgos conducen a considerar que la latitud sur puede presentar una configuración del fenómeno de la sensibilidad estacional que debe ser estudiada en población adulta productiva. Los reportes de su frecuencia y severidad tienden a ser consistentes con la literatura disponible, así como también el señalamiento de su amplitud como fenómeno negativo para la adaptación general de las personas que la experimentan. Su impacto específico en la adaptación laboral continúa siendo un desafío 
pendiente, ya que los estudios sobre esta temática en este ámbito son escasos, si no nulos. La salud laboral debiese considerar la interacción que esta condición climático-geográfica tiene con otros aspectos del trabajo en los que este primer estudio pretende hacer una contribución. En términos prácticos, ampliar el conocimiento de este tema contribuiría a establecer políticas públicas eficientes para prevenir las consecuencias negativas de los cambios lumínicos que se manifiestan tanto en lo físico como en lo mental y afectan tanto el bienestar personal como colectivo de una comunidad.

\section{Referencias}

Adan, A., Archer, S., Loayza, M., Randler, C., Di Milia, L., Natale, V. \& Randler, C. (2012). Circadian typology: a comprehensive review. Chronobiology International, 29(9). doi: 10.3109/07420528.2012.719971

Adan, A., Natali, V. \& Fabris, M. (2006). Propiedades psicométricas de la versión castellana del cuestionario de evaluación de patrón estacional (Seasonal Pattern Assessment Questionnaire, SPAQ). Revista Latinoamericana de Psicología, 38(1), 59-69.

American Psychiatric Association. (2013). Manual Diagnóstico y Estadístico de los Trastornos Mentales DSM-V. Barcelona: Masson.

Angosto, M. P. (1992). Influencia de los ritmos biológicos en el trabajo nocturno. Medicina y Seguridad del Trabajo, 158, 41-46.

Argyle, M. (1992). La psicología de la felicidad. Madrid: Alianza.

Barrientos, J. (2005). Calidad de vida, bienestar subjetivo: una mirada psicosocial. Santiago: Universidad Diego Portales.

Basnet, T. B., Rokaya, M. B., Bhattarai, B. \& Münzbergová, Z. (2016). Heterogeneous landscapes on steep slopes at low altitudes as hotspots of bird diversity in a Hilly Region of Nepal in the Central Himalayas. PLoS One, 11, 1-19. doi: 10.1371/journal.pone.0150498

Bell, H. M. (1934). The Adjustment Inventory. California: Stanford University Press.

Bharvad, M. B. (2015). A study of emotional intelligence and adjustment among school students. The International Journal of Indian Psychology, 2(2), 24-31.

Birchwood, M., Smith, J. O., Cochrane, R., Wetton, S. \& Copestake, S. (1990). The Social Functioning Scale The development and validation of a new scale of social adjustment for use in family intervention programs with schizophrenic patients. The British Journal of Psychiatry, 157(6), 853-859. doi: 10.1192/bjp.157.6.853

Bjorvatn, B., Saxvig, I., Waage, S. \& Pallesen, S. (2020). Self-reported seasonality is strongly associated with chronotype and weakly associated with latitude. Chronobiology International, 38(2), 278-285. doi: 10.1080/07420528.2020.1844725

Bohle, P., Willaby, H., Quinlan, M. \& McNamara, M. (2011). Flexible work in call centers: working hours, work-life conflict y health. Applied Ergonomics, 42(2), 219-224. doi: 10.1016/j.apergo.2010.06.007

Buckby, J. A., Cotton, S. M., Cosgrave, E. M., Killackey, E. J. \& Yung, A. R. (2008). A factor analytic investigation of the Tripartite model of affect in a clinical sample of young Australians. BMC Psychiatry, 8(1), 79. doi: 10.1186/1471-244x-8-79 
Dam, H., Jakobsen, K. \& Mellerup, E. (1998). Prevalence of Winter depression in Denmark. Acta Psychiatrica Scandinavica, 97(1), 1-4. doi: 10.1111/j.16000447.1998.tb09954.x.

Diener, E. (1994). El bienestar subjetivo. Intervención psicosocial. Revista sobre igualdad y calidad de vida, 3(8), 67-113.

Diener, E., Suh, E. M., Lucas, R. E. \& Smith, H. L. (1999). Subjective well-being: Three decades of progress. Psychological bulletin, 125(2), 276.

Fischer, F., Silva-Costa, A., Griep, R., Smolensky, M., Bohle, P. \& Rotenberg, L. (2019) Working Time Society consensus statements: Psychosocial stressors relevant to the health and wellbeing of night and shift workers. Industrial Health, 57(2), 175-183.

Garbazza, C. \& Benedetti, F. (2018). Genetic factors affecting seasonality, mood, and the circadian clock. Frontiers in Endocrinology (Lausanne), 23(9), 481. doi: 10.3389/fendo.2018.00481

Gatón, M., González, M. \& Gaviria, M. (2015). Trastornos afectivos estacionales, "winter blues". Revista Española de Neuropsiquiatría, 35(126), 367-380. doi: 10.4321/S0211-57352015000200010

Goikolea, J. M., Miralles, G., Bulbena-Cabré, A., Vieta, E. \& Bulbena, A. (2003). Adaptación española del cuestionario de evaluación de perfil estacional (Seasonal Pattern Assessment Questionnaire, (SPAQ) en las versiones de adultos e infantojuvenil. Actas Españolas de Psiquiatría, 31, 192-198.

González, M. \& Ibáñez, I. (2018). Propiedades psicométricas de una versión española breve de 30 ítems del Cuestionario de Ansiedad y Depresión (MASQE30). Universitas Psychologica, 17(1).

Goodrich, S. \& Weaver, K. A. (1998). Differences in depressive symptoms between traditional workers and shiftworkers. Psychological Reports. 83, 571-576.

Guérin, J. \& Durrmeyer, G. (1973). Etude de La Fatigue Mentale Industrielle. Institut des Sciences sociales du Travail, Université Paris I, París.

Jiménez, M. I. \& López-Zafra, E. (2011). Actitudes sociales y adaptación social en adolescentes españoles: El papel de la inteligencia emocional percibida. Revista de Psicología Social, 26(1), 105-117. doi: 10.1174/021347411794078417

Joiner, T. E., Catanzaro, S. J., Laurent, J., Sandín, B. \& Blalock, J. A. (1996). Modelo tripartito sobre el afecto positivo y negativo, la depresión y la ansiedad: Evidencia basada en la estructura de los síntomas y en diferencias sexuales. Revista de Psicopatología y Psicología Clínica, 1, 27-34.

Kasper, S., Wehr, T. A., Bartko, J. J., Gaist, P. A. \& Rosenthal, N. E. (1989). Epidemiological findings of seasonal changes in mood and behavior: a telephone survey of Montgomery County, Maryland. Archives of General Psychiatry, 46, 823-833.

Kawasaki, A., Wisniewski, S., Healey, B., Pattyn, N., Kunz, D. Basner, M. \& Münch, M. (2018). Impact of long-term daylight deprivation on retinal light sensitivity, circadian rhythms and sleep during the Antarctic Winter. Scientific Reports, 8, 16185. doi: 10.1038/s41598-018-33450-7

Kegel, M., Dam, H., Ali, F. \& Bjerregaard, P. (2009). The prevalence of seasonal affective disorder (SAD) in Greenland is related to latitude. Nordic Journal of Psychiatry, 63(4), 331-335. doi: 10.1080/08039480902799040 
Kivelä, L., Papadopoulos, M. R. \& Antypa, N. (2018). Chronotype and psychiatric disorders. Current Sleep Medicina Reports, 4(2), 94-103 doi: 10.1007/s40675-018-0113-8

Labbé, M., Veliz, J., Saavedra, I., Arab, E. \& Martorell, B. (2011). Efecto de la variación estacional en el estado de ánimo de adolescentes, estudio prospectivo. Revista Sociedad de Psiquiatría y Neurología de la Infancia y Adolescencia, 22(1), 48-61.

Lambert, G. W., Reid, C., Kaye, D., Jennings, G. L. \& Esler, M. D. (2002). Effect of sunlight and season on serotonin turnover in the brain. The Lancet, 360(9348), 1840-1842. doi: 10.1016/s0140-6736(02)11737-5

Lansdowne, A. T. \& Provost, S. C. (1998). Vitamin D3 enhances mood in healthy subjects during winter. Psychopharmacology, 135(4), 319-323. doi: 10.1007/s002130050517

Lyubomirsky, S. \& Lepper, H. S. (1999). A measure of subjective happiness: Preliminary reliability and construct validation. Social Indicators Research, 46, 137-155.

Lyubomirsky, S. (2008). La ciencia de la felicidad: Un método comprobado para conseguir el bienestar. Barcelona: Urano.

Lyubomirsky, S., Sheldon, K. M. \& Schkade, D. (2005). Pursuing happiness: the architecture of sustainable change. Review of General Psychology, 9(2), 111-131.

Magnusson, A. (1996). Validation of the seasonal pattern assessment questionnaire (SPAQ). Journal of affective disorders, 40(3), 121-129. doi: 10.1016/0165-0327(96)00036-5

Magnusson, A. (2000). An overview of epidemiological studies on seasonal affective disorder. Acta Psychiatrica Scandinavica, 101(3), 176-184. doi: 10.1034/j.16000447.2000.101003176.x

Malbos, D. (2020). Les troubles affectifs saisonniers. Actualités pharmaceutiques, 600, 1922. doi: 10.1016/j.actpha.2020.09.012

Matthews, G. (1998). Morningness-eveningness as a dimension of personality: trait, state, and psychophysiological correlates. European Journal of Personality, 2(4), 277-293.

Nabe-Nielsen, K., Tuchsen, F., Christensen, K., Garde, A. \& Diderichsen, F. (2009). Differences between day and nonday workers in exposure to physical and psychosocial work factors in the Danish eldercare sector. Scandinavian Journal of Work Environment \& Health, 35(1), 48-55. doi: 10.5271/sjweh.1307

Nielsen, H. B., Larsen, A. D., Dyreborg, J., Hansen, A. M., Pompeii, L. A., Conway, S. H., Hansen, J., Kolstad, H. A., Nabe-Nielsen, K. \& Garde, A. H. (2018). Risk of injury after evening and night work-findings from the Danish Working Hour Database. Scandinavian Journal of Work Environment \& Health, 44(4), 385-393. doi: 10.5271/sjweh.3737

Oyane, N., Reidun, U., Pallesen, S., Holsten, F. \& Bjorvatn, B. (2010). Increased health risk in subjects with high self-reported seasonality. PLoS ONE, 5(3), e9498. doi: 10.1371/journal.pone.0009498

Rihmer, Z. (1980). Season of birth and season of hospital admission in bipolar depressed female patients. Psychiatry Research, 3(3), 247-251. doi: 10.1016/01651781(80)90054-2

Roenneberg, T., Pilz, L., Zerbino, G. \& Winnebeck, E. (2019). Chronotype and social jetlag: a (self-) critical review. Biology, 8(54), 1-19. doi: 10.3390/biology8030054

Rohan, K. J., Sigmon, S. T. \& Dorhofer, D. M. (2003). Cognitive-behavioral factors in seasonal affective disorder. Journal of Consulting and Clinical Psychology, 71(1), 22. doi: 10.1037/0022-006x.71.1.22 
Rosen, L. N., Targum, S. D., Terman, M., Bryant, M. J., Hoffman, H., Kasper, S. F. \& Rosenthal, N. E. (1990). Prevalence of seasonal affective disorder at four latitudes. Psychiatry Research, 31(2), 131-144. doi: 10.1016/0165-1781(90)90116-m

Rosenthal, N. E, Carpenter, C. J., James, S. P., Parry, B. L., Rogers, S. L. B. \& Wehr, T. A. (1986). Seasonal affective disorder in children and adolescents. The American Journal of Psychiatry, 143(3), 356-358. doi: 10.1176/ajp.143.3.356

Rosenthal, N. E. (1993). Winter blues: seasonal affective disorder: what it is and how to overcome it. Nueva York: Guilford.

Rosenthal, N. E., Bradt, G. H. \& Wehr, T. A. (1984). Seasonal Pattern Assessment Questionnaire (SPAQ). Bethesda: National Institute of Mental Health.

Rosenthal, N. E., Sack, D. A., Gillin, J. C., Lewy, A. J., Goodwin, F. K., Davenport, Y. \& Wehr, T. A. (1984). Seasonal affective disorder: a description of the syndrome and preliminary findings with light therapy. Archives of general psychiatry, 41(1), 72-80. doi: 10.1001/archpsyc.1984.01790120076010

Rumble, M. E., Dickson, D., McCall, W. V., Krystal, A. D., Case, D., Rosenquist, P. B. \& Benca, R. M. (2018). The relationship of person-specific eveningness chronotype, greater seasonality, and less rhythmicity to suicidal behavior: a literature review. Journal of Affective Disorders, 227, 721-730. doi: 10.1016/j.jad.2017.11.078

Saarijärvi, S., Lauerma, H., Helenius, H. \& Anglé, S. (1999). Seasonal affective disorders among rural Finns and Lapps. Acta Psychiatrica Scandinavica, 99(2), 95-101. doi: 10.1111/j.1600-0447.1999.tb07206.x

Stumpf, W. E. \& Privette T. H. (1989). Light, vitamin D and psychiatry: Role of 1,25dihydroxyvitamin D3 (soltriol) in etiology and therapy of seasonal affective disorder and other mental processes. Psychopharmacol, 97, 285-294.

Tam, E. M., Lam, R. W., Robertson, H. A., Stewart, J. N., Yatham, L. N. \& Zis, A. P. (1997). Atypical depressive symptoms in seasonal and non-seasonal mood disorders. Journal of Affective Disorders, 44(1), 39-44. doi: 10.1016/s0165-0327(97)01447-х

Vendrik, M. (2013). Adaptation, anticipation and social interaction in happiness: An integrated error-correction approach. Journal of Public Economics, 105, 131-149.

Watson, D., Clark, L. A. \& Carey, G. (1988). Positive and negative affectivity and their relation to anxiety and depressive disorders. Journal of abnormal psychology, 97(3), 346. doi: 10.1037/0021-843x.97.3.346

Wehr, T. A., Duncan, W. C., Sher, L., Aeschbach, D., Schwartz, P. J., Turner, E. H. \& Rosenthal, N. E. (2001). A circadian signal of change of season in patients with seasonal affective disorder. Archives of General Psychiatry, 58(12), 1108-1114. doi: 10.1001/archpsyc.58.12.1108

Wirz-Justice, A., Graw, P., Kräuchi, K. \& Wacker, H. R. (2003). Seasonality in affective disorders in Switzerland. Acta Psychiatrica Scandinavica, 108, 92-95. doi: 10.1034/j.1600-0447.108.s418.18.x 
Contribución de los autores: a) Concepción y diseño del trabajo; b) Adquisición de datos; c) Análisis e interpretación de datos; d) Redacción del manuscrito; e) revisión crítica del manuscrito.

I. B. A. ha contribuido en b, c, d; F. L. T. en b, c, d; V. M. C. en b, c, d; C. V. V. en b, c, d; C. J. V. en b, c, d; C. N. E. en c, d, e; C. E-G. en a, b, c, d, e.

Editora científica responsable: Dra. Cecilia Cracco. 